Cahiers de recherches médiévales

\title{
Du rebond parodique
}

Les pièces CLXXIV et CLXXV du Recueil général des jeux-partis français

\section{Patrice Uhl}

\section{(2) OpenEdition}

Journals

Édition électronique

URL : https://journals.openedition.org/crm/5653

DOI : $10.4000 / \mathrm{crm} .5653$

ISSN : 1955-2424

Éditeur

Honoré Champion

Édition imprimée

Date de publication : 20 juin 2008

Pagination : 129-143

ISSN : 1272-9752

\section{Référence électronique}

Patrice Uhl, «Du rebond parodique », Cahiers de recherches médiévales [En ligne], 15 | 2008, mis en ligne le 20 juin 2011, consulté le 15 décembre 2022. URL : http://journals.openedition.org/crm/5653 . DOl : https://doi.org/10.4000/crm.5653 


\title{
酶M
}

\section{Du rebond parodique : les pièces CLXXIV et CLXXV du Recueil général des jeux-partis français}

\begin{abstract}
During the XIIIth century, burghers and trouveres of the North went and theatricalise the fin'amor throughout the jeu-parti. If several pieces of the Recueil général des jeux-partis français betray some sort of "parodic temptation " (the XIV, XV, XVI, XIX, XX pieces and above all the CXXXVIII one), none of them has the radical nature of a parody that can be found in the CLXXIV et CLXXV pieces, entirely inspired by the Sottes Chansons of Ms. Douce 308.

It is in Lorraine, a land usually considered as hardly representative of modern medieval literature, that about 1305-1310 there appears a final attempt at reviving the already somewhat worn-out machinery of the courtly debate. The ambition of the small literary coterie gathered at the court of Jean de Bar around Roland de Reims, no more no less, was, it seems, to ravish the light to the Arras avant-garde while its star was fading. Besides, the same circles in Lorraine are probably also at the origin of the Sottes Chansons of Ms. Douce 308, which inspire, in the same manuscript, the two parodic jeux-partis. This would corroborate the idea of an eastward migration of modern literature at the dawn of XIVth century.
\end{abstract}

Résumé: Tout au long du XIII s., trouvères et bourgeois du Nord se sont amusés à théâtraliser la fin'amor à travers le jeu-parti. Si plusieurs pièces du Recueil général des jeuxpartis français laissent filtrer quelque "tentation parodique " $X I V, X V, X V I, X I X, X X$ et surtout CXXXVIII), aucune ne possède le caractère de parodie radicale des pièces CLXXIV et $C L X X V$, qui se nourrissent intégralement des Sottes Chansons du Ms. Douce 308.

C'est en Lorraine, terre d'ordinaire jugée peu représentative de la modernité littéraire au Moyen Âge, que, vers 1305-1310, se localise cette tentative extrême de relancer de l'intérieur la machinerie passablement épuisée du débat courtois. L'ambition du petit cénacle réuni à la cour de Jean de Bar autour de Roland de Reims paraît, ni plus ni moins, avoir été de ravir le flambeau à l'avant-garde arrageoise au moment où l'étoile de celle-ci déclinait. Il est du reste probable que les mêmes milieux lorrains soient également responsables des Sottes Chansons du Ms. Douce 308, dont se nourrissent, dans le même manuscrit, les deux jeuxpartis parodiques. Ce qui conforterait l'idée d'un déplacement de la modernité littéraire vers l'Est au début du XIV siècle.

Dans Burlesque et obscénité chez les troubadours, Pierre Bec définit le «contre-texte» en ces termes :

[Le contre-texte] n'est pas ambigu. Il s'installe en effet dans le code littéraire, utilise ses procédés jusqu'à l'exaspération, mais le dévie fondamentalement de son contenu référentiel. Il n'y a donc pas d'ambiguïté à proprement parler, mais juxtaposition à des fins ludiques et burlesques d'un code littéraire donné et d'un contenu marginal, voire subversif. Le code textuel endémique reste donc bien l'indispensable référence, fonctionne toujours dans la plénitude de ses moyens, mais à contre-

Cahiers de Recherches Médiévales, 15, 2008 
courant. [...]. Le contre-texte est donc, par définition, un texte minoritaire et marginalisé, une sorte d'infra-littérature (underground). Sa référence paradigmatique reste le texte, dont il se démarque, et son récepteur, inévitablement le même que celui du texte. Car sa réception et son impact sont étroitement liés aux modalités du code textuel majoritaire. ${ }^{1}$

Dans les limites de cette définition, les pièces CLXXIV et CLXXV du Recueil général des jeux-partis français ${ }^{2}$ sont de plein droit à considérer comme des «contre-textes»; elles s'installent en effet dans le code d'une sub-tradition constituée de longue date, et dont la textualité nous est parvenue à travers un grand nombre de chansonniers ou fragments de chansonniers: $A, C, D, E, G, H^{a_{3}}, I, K, M$, $N, O, P, Q, R, S, T, U, V, W, X, Y, Z, a, b, c$ et Cambrai 1328; une générosité qui en dit long sur la faveur dont cette forme de poésie a joui tout au long du XIII ${ }^{\mathrm{e}}$ siècle, et même un peu au-delà ! Mais l'intérêt de ces pièces réside avant tout dans le fait qu'elles redoublent la parodie du «code textuel majoritaire» (le jeu-parti), en se nourrissant du corpus de l'anti-canso courtoise (la sotte chanson) ${ }^{4}$. Autrement dit, elles instaurent une double distance ironique par rapport aux usages poétiques courtois, moquant à la fois le jeu dialogique théâtralisant la fin' amor et, par rebond, la forme dont ce jeu est issu: le grans chans. Cette mise en abyme invite à s'interroger sur l'enjeu poétique réel d'une telle parodie, intervenant au crépuscule d'une riche sub-tradition dérivée du partimen occitan et dans une région jugée d'ordinaire marginale du point de vue de la «modernité » littéraire : la Lorraine ${ }^{5}$.

${ }^{1}$ P. Bec, Burlesque et obscénité chez les troubadours. Le contre-texte au Moyen Âge, Paris, Stock, 1984, p. 11-13.

2 A. Långfors, A. Jeanroy et L. Brandin, Recueil général des jeux-partis français, Paris, Champion (SATF), 1926, t. II, p. 275-277 et p. 278-281. Je n'ai malheureusement pas eu accès à l'ouvrage récemment publié par Mary Atchison, The Chansonnier of Oxford Bodleian MS Douce 308 : Essays and Complete Edition of Texts, Aldershot, Ashgate, 2005.

${ }^{3} \mathrm{H}^{\mathrm{a}}=$ La Haye, Koninklijke Bibliotheek, dossier $131 \mathrm{D}$ I; voir R. Crespo, «Un 'jeu-parti' inedito », Studi Medievali, serie terza, 23, 1982, p. 957-969.

${ }^{4} \mathrm{Cf}$. L. Hutcheon, «Ironie, satire, parodie. Une approche pragmatique de l'ironie », Poétique, 46, 1981, p. 140-155; ici p. 143 : «La parodie n'est pas un trope comme l'ironie : elle se définit normalement non pas en tant que phénomène intratextuel mais en tant que modalité du canon de l'intertextualité. Comme les autres formes intertextuelles (telles que l'allusion, le pastiche, l'imitation et ainsi de suite), la parodie effectue une superposition de textes. Au niveau de sa structure formelle, un texte parodique est l'articulation d'une synthèse, d'une incorporation d'un texte parodié (d'arrière-plan) dans un texte parodiant, d'un enchâssement du vieux dans le neuf. Mais ce dédoublement parodique ne fonctionne que pour marquer la différence: la parodie représente à la fois la déviation d'une norme et l'inclusion de cette norme comme matériau intériorisé. »

${ }^{5}$ Cf. J. Kooijman, Trouvères lorrains. La poésie courtoise en Lorraine au XIII ${ }^{e}$ siècle, s.l., Seurat, 1974. Cette anthologie bilingue accorde une place très importante aux jeux-partis du chansonnier $I$ (p. 29-75). 
La tentation parodique couve dans les jeux-partis dès l'époque la plus faste du genre. On voit mal en effet comment cette « forme 'jouée' du chant courtois » eût pu échapper au rire des moqueurs, vu que, au Moyen Âge, toute sub-tradition littéraire, quasiment du temps où elle émergeait, a suscité sa propre contestation ${ }^{7}$ : la chanson de geste a produit Audigier, le grand chant la sotte chanson, la Psychomachie la bataille allégorique burlesque (Enfer et Paradis, Caresme et Charnage) ${ }^{8}$, le "devinalh» des contrefaçons septentrionales (Besturné, Malade sui de joie espris, Oez com je sui bestornez... $)^{9}$, etc. S'il n'existe, comme l'observe Michèle Gally, "qu'un seul jeu véritablement parodique ${ }^{10}$ parmi les jeux-partis arrageois (section D du livre de Långfors-Jeanroy-Brandin) - la pièce CXXXVIII, où Gillebert de Berneville pose à Thomas Herier la question suivante : "Renonceriez-vous à tout jamais aux pois au lard, en échange de la fortune d'Audefroi Louchart?»-, tel n'est pas le cas dans le chansonnier $R$, où plusieurs unica sont de tour ouvertement parodique ; par exemple (je m'en tiens aux demandes) :

XIV (Jehan d'Estruen à Colart le Changeur) : «Deux dames ont parié : l'une qu'elle me tirerait les cheveux, l'autre qu'elle me serrerait le cou. Laquelle doit, de préférence, être mon amie ?»

XV (Sandrart Certain à Colart [le Changeur?]) : «De deux amoureux, du reste égaux en mérite, qui adressent leurs vœux à la même femme, l'un devient aveugle l'autre sourd. Lequel a le plus de chances de réussir?»

XVI (un «Sire» à Sandrart Certain): «Si vous étiez comme moi, 'profès en dévotion', et appelé à fréquenter nonnes et béguines, préféreriez vous courtiser une nonne ou une béguine?»

XIX (Hue à Robert [le Duc]) : «Je ne puis recouvrer les bonnes grâces de celle que j'aime, si je ne lui donne de grands coups. Me conseillez-vous de le faire?»

XX (Jehan [d'Estruen ?] à Robert [le Duc ?]) : «J'aime une dame qui a plus de soixante ans, et qui ne veut m'accorder son amour à moins que je ne lui jure de n'en jamais aimer une autre tant qu'elle vivra. Que dois-je faire?»

Le jeu-parti se caractérise d'abord par son caractère ludique : «Le jeu parti est une polémique pour rire, un jeu de société. Il ne parle ni vrai, ni faux, ni bien, ni

\footnotetext{
${ }^{6}$ M. Gally, «Disputer d'amour : les Arrageois et le jeu-parti », Romania, 107, 1996, p. 55-76; ici, p. 76.

${ }^{7}$ Cf. P. Zumthor, Essai de poétique médiévale, Paris, Seuil, 1972, p. 104-106.

${ }^{8} \mathrm{Cf}$. P. Uhl, La constellation poétique du non-sens au Moyen Âge. Onze études sur la poésie fatrasique et ses environs, Paris, L'Harmattan et Université de La Réunion, 1999, p. 63-71.

${ }^{9}$ Cf. P. Uhl, «Compositions de oppositis d'oc et moqueurs d'oïl», Poésie et rhétorique du non-sens. Littérature médiévale et littérature orale, dir. S. Mougin et M.-G. Grossel, Reims, Presses Universitaires de Reims, 2004, p. 63-87.

${ }^{10}$ M. Gally, art. cit., p. 75. La pièce CXL (Recueil général, t.II, p. 157-161), entre Gillebert de Berneville et Amors, sur le rapport entre pilosité et amour ne me parait pas non plus dépourvue d'arrière-pensées parodiques, en dépit des explications rationalisantes des éditeurs (Recueil général, t.I, p. IX-X).
} 
mal, mais il évacue, ainsi, l'enjeu poétique et éthique de la poésie courtoise $»^{11}$. Dans les puys du Nord, où se «couronnaient» avec autant d'honneur les chansons d'amour et les sottes chansons contre Amour, l'homogénéité idéologique de la fin'amor présentait de nombreuses lézardes. Des raisons socio-poétiques (proximité des publics, émergence de nouvelles valeurs liées au patriciat urbain, etc.) l'expliquent en large part. En schématisant, le vieil «idéalisme» courtois, modèle longtemps respecté dans les cités du Nord, dut, au fil du temps, composer avec de nouvelles représentations plus directement ancrées dans le «réalisme» bourgeois. Issu de la chanson courtoise, le jeu-parti s'en distingue toutefois par sa liberté vis-àvis des thématiques convenues; d'où "de fréquentes rencontres entre un discours courtois et un discours volontiers prosaïque ${ }^{12}$; d'où l'intrusion de la vie courante, du quotidien, du trivial dans le discours amoureux. On ne peut que donner raison à Michèle Gally, lorsqu'elle écrit: «L'émergence de ces nouveaux champs de représentations [...] n'est-elle pas aussi l'indice d'un essoufflement, et d'une insuffisance des représentations traditionnelles? Plus, en effet, qu'un simple enrichissement du vocabulaire ils modifient, à terme, le discours lyrique attendu $»^{13}$. De fait, au sein du «registre aristocratisant $»^{14}$, le lien entre le jeu-parti et le grand chant des trouvères a, semble-t-il, toujours été beaucoup plus lâche qu'entre le partimen et la canso des Occitans. Encore que chez les troubadours, le dialogisme formât souvent une modalité élective de contestation de la doxa courtoise... ${ }^{15} \mathrm{Jeu}$ de société, sans réelle implication «doctrinale», le jeu-parti offrait en tout cas aux poètes des possibilités de variations registrales que le grand chant n'autorisait théoriquement pas. Mais il arriva un temps où le jeu finit par tourner à vide: ressassées depuis des lustres, les questions amoureuses peinaient à se renouveler; les sujets dilemmatiques s'épuisaient inexorablement (plusieurs pièces du recueil de Långfors posent du reste la même question). La casuistique amoureuse possédait des limites que l'ingéniosité des poètes ne parvenait plus à contourner. Il fallait donc ouvrir le jeu à de nouvelles possibilités, à de nouvelles règles. En déplaçant les bornes $\mathrm{du}$ "courtoisement correct», le rire, le gros rire, représentait dans ces conditions un moyen commode de dépassement.

La tentation parodique, qui affleure dans les jeux-partis arrageois et se fait plus pressante dans les unica du chansonnier $R$, débouche au début du $\mathrm{XIV}^{\mathrm{e}}$ siècle sur un type de parodie radicale illustré par les pièces CLXXIV et CLXXV du chansonnier I. Ces deux poèmes opposent, le premier Quaré à Roland de Reims ; le

\footnotetext{
${ }^{11}$ M. Gally, art. cit., p. 75.

${ }^{12}$ Ibid., p. 67.

${ }^{13}$ Ibid., p. 69.

${ }^{14} \mathrm{Cf}$. P. Bec, La lyrique française au Moyen Âge (12 $-13^{e}$ siècles). Contribution à une typologie des genres poétiques médiévaux, Paris, A. \& J.Picard, 1977, vol.I : Études, p. 33-43.

${ }^{15} \mathrm{Cf}$. P. Uhl, «Du casus amoris : «Peut-on corner au cul d'une dame? » à la tenso obscène entre Montan et la Domna - l'anti-doxa courtoise en dialogue », Les représentations de la déviance, dir. C. Duboin, Paris, L'Harmattan et Université de la Réunion (Cahiers CRLH, 13), 2005, p. 93-102.
} 
second Roland de Reims à Aubert (ou Aubertin); ils occupent dans la troisième section du chansonnier (la section réservée au genre) les $19^{\mathrm{e}}$ et $20^{\mathrm{e}}$ rangs $^{16}$ :

\section{CLXXIV}

«Rollans, amins, au fort me consilliés.

J'ain maugrei moi et de bone saixon :

J'ain la Noire, la feme Souleis Viés,

Et elle dit ke je n'i ai raison,

K'elle aimme mués Railart Tiretacon,

Un sien sergent, ke li bet sa buee.

Lais! Je l'ain tant, j'en ai la pance enflee,

Mais je n'i puis pour argent avenir.

Dites, Rollant, l'avrai je por teusir?

- Par Deu, Quareis, je suis toz ranbraisiés

De respondre, mais j'ai mal au talon.

Bien ait vos cuers cant il c'est hireciés

Por dame amer de si gente faison.

C'ai droit bordeir, plus belle ne seit on.

Ce n'est mie san[s] sans ne sans testee

Ke vos l'aveis si fort enamouree.

Mais c'elle avoit de vo cul un so[s]pir,

Ces cuers poroit bien a vostre flaitir.

- Rollans, amins, ne suis pas bien aixiés

De mes amors doner si riche don.

Je vorroie estre en vin d'Ausai noieis

Et de s'amor ne me fust un bouton.

Lairai l'amer? Oiil voir. Je voir non.

Se d'un sospir je l'avoie avantee

Pour la table détaillée des pièces des six sections, cf. G. Steffens, "Die altfranzösische Liederhandschrift der Bodleiana in Oxford, Douce $308 »$, Archiv für das Studium der neueren Sprachen und Literaturen, 104, 1900, p. 331-354; spéc., p. 346-354. Le chansonnier $I$ est connu pour ses unica: section 1 : Grands chants (91 pièces, dont 39 unica) ; section 2 : Estampies (19 pièces, dont 17 unica) ; section $3:$ Jeux-partis (38 pièces, dont les 26 unica autour de Rolant au début et à la fin de la section : 3.1-3.21 et 3.32-3.36; 3.22-3.23 conservent trente demandes d'amour; une douzaine de jeux-partis attestés ailleurs sont insérés en $3.24-27,27 \mathrm{a}, 28,28 \mathrm{a}, 29,29 \mathrm{a}, 30-31$ et $31 \mathrm{a}$ ); section 4 : Pastorelles (38 unica); section 5: Ballettes 165 unica); section 6 : Sottes chansons (22 unica d'après la table). La dernière partie du chansonnier, qui ne comporte pas de rubrique, contient 74 motets, dont 57 unica, et 37 rondeaux, dont 35 unica. Cf. M.-R. Jung, «Les formes strophiques des jeux-partis autour de Rolant (chansonnier d'Oxford, Bodleaian Library, Douce 308)», et multum et multa. Festchrift für Peter Wunderli zum 60. Geburtstag, Herausgegeben von E. Werner, R. Liver, Y. Stork, M. Nicklaus, Tübingen, Gunter Narr Verlag, 1998, p. 387-398 ; spéc., p. 387-389. 
Ki vient de bais, elle seroit ferdee :

Rois, contes, dus la feroient ravir,

Moi covanroit après s'amor glaitir.

- Biaus dous Quairez, point jallous n'an soiez

C'on la vos doie embleir en traïxon.

Nuns ne poroit lou jor estre haitiés

Ke la vairoit lou main en sa maison,

Cant elle siet leis son feu de charbon,

Noire biauteit l'ait si enluminee ;

Ce vos l'ameis, c'est dure destinee.

Conforteis vous, je li oï jehir :

Por vostre amor ne puet son vant tenir.

- Por vos biaus mos, Rollans, suix enjaiez

Si con je fuxe asomeis d'un premon.

Je vos croirai, mais ju ai les .ij. piés

Toz enjalleis et s'ai la çurkeuson

Por li amer. C'est en confession

Ke je vos di, si soit chose cellee :

Por ceu l'ain ju k'elle est trop mal buee,

Et s'aimme mués totans a chans crupir

K'elle me faice en un biau leit gesir. »

«Roland, mon ami, tout au moins conseillez-moi. J'aime malgré moi et ce bien à propos : j'aime la Noiraude, la femme de Vieux Souliers, et elle dit que ce n'est pas raisonnable, car elle aime mieux Railart le Rapiéceur, un de ses valets qui excelle au battoir $^{17}$. Hélas! je l'aime tant que j'en ai la panse tout enflée, mais je ne puis obtenir son amour contre argent. Dites, Roland, y parviendrai-je en toussant?

- Par Dieu, Quaré, je suis tout excité de vous répondre, mais j'ai mal au talon. Quel bonheur pour votre cour ${ }^{18}$ de s'être ainsi ébouriffé d'amour pour une dame de si noble façon! Pour dire une belle bourde ${ }^{19}$, on ne saurait rencontrer plus belle femme. Ce n'est certes pas sans raison ni sur un coup de tête que vous vous en êtes

\footnotetext{
${ }^{17}$ Batre sa buee renvoie bien sûr à la lessive, au linge que l'on bat pour le nettoyer : Il ouyt femmes qui batoient une buee (Perceforest). Mais le verbe batre, au sens de «foutre «, entre dans une foule de locutions ; cf. Gunnar Tilander, Lexique du Roman de Renart, Paris, Champion, 1971, p. 21, s.v. BATRE.

${ }^{18}$ J'ai traduit cuers par «cœur», mais cuer peut être aussi une forme lorraine pour cuir "peau », comme dans la sotte chanson III, $5:[\ldots]$ s'an suix an teil frixon/ C'a poc n'an pert lou cuer de mon talon.

${ }^{19}$ Je ne pense pas que bordeir soit "pour behorder, au sens grivois" (Recueil général, t. II, p. 277, n. au v.14). Behorder se rencontre en ce sens dans CLXXV, 22 ; border "dire des bourdes » apparait dans la sotte chanson VII, 40 : Flors estes de cortoisie et de cens, a droit border.
} 
entiché. Mais si elle obtenait de votre cul un soupir, son cœur pourrait bien se jeter sur le vôtre.

- Roland, mon ami, cela ne me plaît guère de donner de mon amour un si riche gage. Je voudrais être noyé dans le vin d'Alsace et que son amour ne comptât pour moi pas plus qu'un simple bouton. Renoncerai-je à l'amour? Oui, certes. Mais non, pas du tout. Si d'un soupir venu de mon cul je l'avais éventée, elle en serait toute fardée : rois, comtes, ducs me la feraient enlever et il ne me resterait plus qu'à glapir de désespoir.

- Mon très cher Quaré, ne soyez pas jaloux qu'on cherche à vous l'enlever par trahison. Nul qui la verrait le matin dans sa maison ne pourrait être en forme de la journée : quand elle s'assied auprès de son feu de charbon, elle est toute barbouillée d'une noire beauté. Si vous l'aimez, c'est là une bien rude destinée ! Mais consolezvous, je l'ai entendue avouer: du fait de votre amour, elle ne sait aucun vent contenir. $^{20}$

- Grâce à vos bonnes paroles, Roland, me voici aussi réjoui que si j'avais été assommé d'un coup de gourdin ${ }^{21}$. Je vous croirais volontiers, mais à cause de l'amour que je ressens pour elle, j'ai les deux pieds engourdis et brûlants de démangeaisons ${ }^{22}$. C'est en confession que je vous le dis, que cela reste entre nous : je l'aime, parce qu'elle est fort mal lavée et parce qu'elle préfère de loin s'accroupir dans les champs plutôt que de me faire coucher dans un bon lit. »

\section{CLXXV}

«Concilliez moi, Aubertin, je vos prie ;

D'Amors saveis tant c'on en puet songier.

J'ain et desir, mais j'ai teile chosie

Ke nuns for[s] moi n'aimmet ne ne tient chier.

Sovant me fait por s'amour rechaignier

Et dexirer ma povre haraudie,

Car li biauteit ki est de li torchie

Et ces gens cors pareis de meschëance

M'ont si tolut et sant et contenance

K'il me covient de joie regeteir.

Dites, conpain, vient ceu de bien amer?

- Rolans, biaus niés, j'ai lut la berkenie

De chief an chief por vos aconcillier.

D'Amors vos vient celle grant raverie

Ke vos faites por la belle a vis fier.

Servez la bien ; a moins por souhaidier

\footnotetext{
${ }^{20} \mathrm{Il}$ y a peut-être un jeu de mots sur vant « vantardise » et vant « vent, pet ».

${ }^{21}$ Cf. Recueil général, t.II, p. 277, n. au v.38: «Premon. Instrument inconnu». On a rapproché premon du wallon prime "perche, longue pièce de bois ».

${ }^{22}$ Cf. ibid., n. au v. 40 : «Çurkeuson pour surcuisson ? Ce mot, non attesté, pourrait signifier 'vive démangeaison'; voy. Littré, CUISSON, $\mathrm{n}^{\circ} 2$ ». Keus «cuits» se rencontre dans la sotte chanson I, 39 : boutons mal keus...
} 
Li Rois des [C]iaus vous ferait belle aïe.

Faites vo dame une grant cortoixie

De la cowe d'une viés treue rance.

C'elle ait la tous, se serait grant pitance ;

Se par teil don la poiez enherber,

Ancor poriés ver[s] son val behorder.

- Aubert, bien voi vos aveis acointie

La vïande dou boin conte Adegier,

Cant ma dame, qui est dure enemie

Voleis faire true rance maingier.

Cant je lai voi crupir sor son fumier

Et je li di : " Deu te saut, belle amie »,

Par fine amor me fait une reupie

Et dont sorit ; par droite desperance

Ver[s] li m'an voix, les brais a col li lance;

Cant je la voil catillier et ribeir,

Com singesce la vairiez berbeteir.

- Rollans, amins, de sa barbeterie

La deveis vos durement gracier.

Soiez bien liés et meneis bone vie

Si c'on vos dust trestous les dens raier.

Rollan[s], por li vos faites escorchier,

Tant vos aimme k'elle an serait trop lie ;

La gent diront : «C'est por melancolie»

Et c'i vos blasme, dites : «C'est par anfance».

Ne cuit k'il ait nul clerc an toute France

Ke vos peüst millor consoil doner,

$\mathrm{Ce}$ vos dous eus ne li faites crever.

- Vostre consoil, A[u]bert, ne me plait mie :

Wardeis lou bien, il vos avrait mestier.

Car ma dame, qui est de sans farcie,

M'ait jai bien dit k'elle m'aimme des ier.

Mais, tant i ait, can la doi aprochier,

Si sotement la voi envisagie

Ke por s'amor me prant la furnexie.

Adont pri Deu ke j'an parte a vitance,

Mais elle dit ke me ferait despance

De son gent cors, se la voil escoler,

Et je li di : «lairiés moi enivrer?»

«Conseillez-moi, Aubertin, je vous en prie ; vous savez plus de choses sur Amour qu'on ne peut en rêver. J'aime et je désire une dame, mais j'en ai choisi une que personne, sauf moi, ne saurait aimer ni apprécier. Souvent, d'amour, elle me fait braire comme un âne et déchirer ma pauvre souquenille, car la beauté dont elle est 
torchée ${ }^{23}$ et son joli corps dépourvu de tout attrait m'ont tellement ôté sens et contrôle de moi qu'il me faut ruer de joie. Dites, compagnon, est-ce là un des effets de l'amour?

- Roland, mon cher neveu, j'ai étudié le «Traité des Bergers $»^{24}$ de bout en bout pour vous conseiller. C'est d'Amour que vous vient la grande folie dont vous souffrez à cause de la belle au farouche visage. Servez-la bien; tout au moins dans vos souhaits, le Roi des Cieux vous accordera une aide précieuse. Faites à votre dame une grande faveur en lui offrant la queue d'une vieille truie rance. Si elle en attrape la toux, ça sera un grand soulagement. Si, par un tel don, vous parveniez à l'ensorceler, alors vous pourriez jouter du côté de son petit val.

- Aubert, je vois bien que vous avez du goût pour la nourriture du bon comte Audigier, puisque vous voulez faire manger à ma dame, qui est une rude diablesse, de la truie rance. Quand je la vois s'accroupir sur son fumier et que je lui dis : « Dieu te sauve, belle amie ! », par fin' amour elle me fait un rot, ce qui la fait sourire ; fou de désespoir, je cours vers elle et lance les bras autour de son cou. Mais quand je la veux caresser et cajoler, vous la verriez grommeler comme une guenon...

- Roland, mon ami, vous devez grandement la remercier de ses grommellements. Soyez heureux et prenez du bon temps, comme si on devait vous arracher toutes les dents. Roland, pour elle, faites-vous écorcher vif; elle vous aime tant que ça la mettra en joie. Les gens diront: "C'est par mauvaise humeur», mais si quelqu'un vous blâme, répondez: "C'est par enfantillage ». Je ne pense pas qu'il y ait un seul clerc dans toute la France qui puisse vous donner un meilleur conseil, à moins que vous ne vous fassiez crever les deux yeux par elle.

- Votre conseil, Aubert, ne me plaît guère : gardez-le pour vous, il vous sera utile un jour. Car ma dame, qui est pleine de bon sens, m'a dit hier qu'elle m'aimait. Pour autant, quand je dois m'approcher d'elle, je lui vois une si sotte expression au visage que, pour l'amour de ma dame, la frénésie me reprend. C'est pourquoi, à mon grand déshonneur, je prie Dieu de m'en séparer. Mais elle dit qu'elle me fera des prodigalités de son joli corps si je veux la prendre par le cou, alors je lui réponds : «Me laisserez-vous m'enivrer? $»^{25}$

${ }^{23}$ Torchier «torcher» ou torsier, trosser «charger»? Le même verbe est employé dans une sotte chanson : Por la biauteit dont vos cors est torchiés (VII, 27). Cf. Recueil général, t. II, p. 280, n. au v.7: «Faut-il traduire : 'La beauté qui est absente d'elle'?»; ce que gloserait le vers $8:$ Et ces gens cors pareis de meschëance («chose de nulle valeur»).

${ }^{24}$ Berkenie est repris sous le lemme Bergerie dans G. Lavis et M. Stasse, Lexique des jeuxpartis. Concordances et index (d'après l'édition d'A. Långfors), Liège, Faculté de Philosophie et Lettres de l'Université de Liège, 1995 ; bergerie « manuel des vétérinaires pour moutons » (T-L, I, 926, s.v. BERGERIE). D'après G. Lubinski, «Die Unica der Jeux-Partis des Oxforder Liederhandschrift», Romanische Forschungen, 22, 1908, [p. 506-598], p. 591, le mot signifierait «calendrier». Il est du reste question d'un calendrier dans une sotte chanson : Mais j'ai trovei dedens mon callendier/ C'amer la doi par devers les trumiaus (XXII, 8-9).

${ }^{25}$ La note au vers 55 du Recueil général (II, 281) se réduit à une question : «Sens? ». C'est despance $=$ despense "vinasse, piquette » au vers 53 qui éclaire la pointe finale ; voir : Tu mens, ce n'est mie despense,/ Ainz est bon vin (Dit des Traverses, 96). Le jeu de mots est intraduisible. 
Les liens entre ces deux pièces et les sottes chansons du manuscrit d'Oxford ont été remarqués depuis longtemps ${ }^{26}$. Arthur Långfors est le premier à avoir listé les «concordances verbales» les plus frappantes entre les pièces des deux genres, dans l'introduction de son édition des recueils de sottes chansons ${ }^{27}$. Une dizaine de pièces sont concernées : I (Chans de singe ne poire mal pellee), II (Amors graci de son joli presant), III (Bien doit chanter qui est si fort chargiés), IV (Chanteir m 'estuet jusc'a jor dou juïse), V (Quant j'ö crïer rabardie), VI (Quant voi vendre char de porc soursamee), VII (Quant j'oi la quaile chausie), VIII (Quant an yver voi ces ribaus lancier), XV (Quant voi ploreir lou froumaige on chazier), XVI (Quant voi paroir la parselle on vert bleif) et XXII (Devant aost, c'on doit ces bleif soier) ; soit plus de la moitié des poèmes intégralement conservés ${ }^{28}$. Mais si toutes les sottes chansons n'ont pas été exploitées (n'oublions pas que quatre pièces ont été perdues et que deux autres nous sont parvenues dans un état lacunaire), il est douteux que Roland et ses partenaires n'aient pas connu le reste de la collection.

Voici, à l'échelle des trois premiers couplets du jeu-parti CLXXIV, quelquesunes de ces concordances (j'ai à l'occasion étoffé le relevé de Långfors) :

\begin{tabular}{|c|c|c|}
\hline CLXXIV, 2-4 & $\leftrightarrow$ SC III, 1-3 & $\begin{array}{l}\text { Bien doit chanter qui est si fort chargiés } \\
\text { De bone amor con je sui sans raison. } \\
\text { J'ain la feme Souleis Viés Houziaus Viés. } \\
\text { Je l'ain forment et de bonne saixon. }\end{array}$ \\
\hline CLXXIV, 5-6 & $\leftrightarrow$ SC II, 22-24 & $\begin{array}{l}\text { Et elle dit : « J'ain moult millour de vous : } \\
\text { Nostre bergier c'ait la teste plumee, } \\
\text { Ke l'autre soir m'estordit ma buee. }\end{array}$ \\
\hline CLXXIV, 7 & $\begin{array}{l}\leftrightarrow \text { SC I, 29-30 } \\
\leftrightarrow \text { SC VI, } 3\end{array}$ & $\begin{array}{l}\text { Vostre biauteit se vint an moi flaitir } \\
\text { Si asprement j'an ai la pance anflee. } \\
\text { De bone amor ai si la pance enflee. }\end{array}$ \\
\hline CLXXIV, 8-9 & $\begin{array}{l}\leftrightarrow \text { SC I, } 40 \\
\leftrightarrow \text { SC XV, } 19 \\
\leftrightarrow \text { SC II, } 38\end{array}$ & $\begin{array}{l}\text { Tot ceu en boins, a vostre eus, por tusir. } \\
\text { Si ke, can celle por cui je n'os tusir... } \\
\text { C'a main vos voit, lou soir an ait la tous... }\end{array}$ \\
\hline CLXXIV, 10- & $11 \leftrightarrow$ SC III, 5 & C'a poc n'an pert lou cuer de mon talon... \\
\hline CLXXIV, 12 & $\begin{array}{l}\leftrightarrow \text { SC III, } 8 \\
\leftrightarrow \text { SC VI, } 21\end{array}$ & $\begin{array}{l}\text { Cant je la voi, d'amors suix hereciés } \\
\text { Dont me comance a hiricier li piaus... }\end{array}$ \\
\hline
\end{tabular}

${ }^{26}$ Cf. G. Lubinski, op. cit. (1908).

${ }^{27}$ A. Långfors, Deux recueils de sottes chansons : Bodléienne Douce 308 et Bibliothèque Nationale, fr. 24432, Helsinki, Suomalainen Tiedeakatemia (Annales Academice Scientiarum Fennicce, B LIII, 4), 1945, p. 15-21, chapitre «Les sottes chansons modèles de jeux-partis ».

${ }^{28}$ D'après l'abecedaire du chansonnier, la section 6 comportait à l'origine 22 pièces ; à la suite d'une mutilation entre les feuillets 253 et 254 actuels, quatre pièces ont été perdues : X, XI, XII et XIII ; les pièces IX et XIV sont lacunaires, l'une de la fin, l'autre du début. 


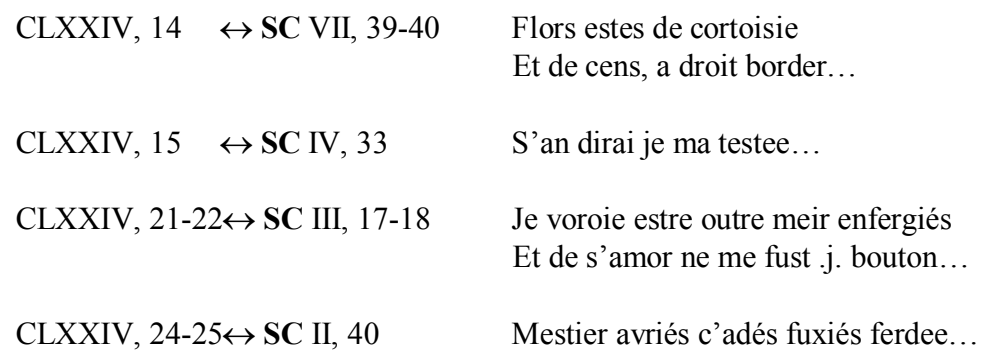

On comprend tout de suite, d'après cet échantillon, qu'aucune sotte chanson particulière n'a été à la source de l'un ou l'autre des jeux-partis, ni même de l'un ou l'autre des couplets ; les Sottes Chansons, prises comme un tout, forment l'architexte de référence. Les parodies de la section 6 constituent une sorte de réservoir paradigmatique dans lequel les partenaires des deux jeux-partis ont puisé des segments phrastiques, des formules, des mots, éventuellement des «thèmes» ou des «motifs»; des éléments qu'ils ont ensuite réemboîtés à leur guise au sein des couplets, se bornant à homogénéiser métriquement et syntaxiquement le collage, et à lui donner le tour argumentatif seyant au genre moqué. Conformément à la règle d'or de toute parodie, le formalisme du modèle a été impeccablement respecté. Rien sous cet angle ne distingue un jeu-parti parodique d'un jeu-parti ordinaire. Dans ces deux pièces, le casus à débattre est exposé, comme il se doit, dans le premier couplet, lequel débute par une adresse au partenaire (CLXXIV : « Rollans, amins, au fort me consilliés »; CLXXV : «Concilliez moi, Aubertin, je vos prie ») et se clôt sur la question à trancher (CLXXIV: «Dites, Rollant, l'avrai-je en toussant? "; CLXXV : «Dites, conpain, vient ceu de bien amer?»); le débat s'engage ensuite dans le respect scrupuleux des conventions langagières et des préséances du dialogue «courtois» (CLXXIV : - Par Deu Quareis.../-Rollans, amins..../-biaus dous Quairez.../ Por vos biaux mos, Rollans... ; CLXXV :-Rolans, biaus niés.../_ Aubert... /-Rollans, amins.../-Vostre consoil, Aubert...). Abstraction faite du contenu, ces deux poèmes ne se signalent par aucun écart ; le «code textuel endémique» n'est jamais violé. Pourtant il s'agit d'un jeu dans le jeu, consistant à "farcir» une grille strictement formalisée (génériquement, typologiquement, discursivement...) de bourdes tirées d'un corpus (contre-)textuel préexistant, et déjà traditionalisé. Du coup, l'invention reste de bout en bout tributaire des modèles créés par les parodistes du grans chans: ridiculisation des effets de l'amour, exagérations burlesques, plongées occasionnelles dans le non-sens, équivoques obscènes, scatologie, plaisanteries alvines, etc. Mais pour que ce jeu dans le jeu conservât pertinence et efficacité, pour que n'existe aucune ambiguïté dans le décodage, il faut supposer entre les joueurs et le public une totale connivence ; autrement dit, il faut supposer que les sottes chansons qui inspirèrent Roland et ses partenaires étaient aussi familières au public qu'à eux-mêmes.

Mais quel était ce public? Dans son étude linguistique des sottes chansons du chansonnier I, Långfors remarquait que «la langue de l'auteur (ou des auteurs) n'était pas le 'picard' banal qui se relève dans la majeure partie des productions littéraires du nord de la France»; plus loin il précisait : «Mais ces particularités ne 
permettent pas une localisation exacte de nos chansons ni ne sauraient en aucun cas exclure les grands centres picardo-artésiens ${ }^{29}$. Selon lui, donc, les sottes chansons pouvaient très bien avoir vu le jour dans une autre région que celle où le manuscrit avait été composé et exécuté («dans l'est de la France»). Ce qui, dans l'absolu, est une position défendable. Une partie des pièces de la section 3 du chansonnier, par exemple, est d'origine arrageoise : les trente demandes d'amour (3.22 et 3.23), sont presque toutes reconductibles à des questions débattues dans l'entourage de Jean Bretel, de même que les jeux-partis transcrits à leur suite (3.24-3.31a)... Mais il en va tout autrement pour les unica auxquels Roland de Reims est associé : leur provenance est clairement lorraine, et selon toute apparence barroise.

Contrairement aux sottes chansons (toutes anonymes), ces unica sont signés : les noms des partenaires et souvent des juges nous sont en effet restés. Ce qui ne veut pas dire que nous soyons parfaitement renseignés sur les uns et les autres, ni que la signature ne masque pas à l'occasion un anonymat de fait: Roland de Reims, par exemple, qui joue à la manière de Jean Bretel le rôle de chef de file, reste un parfait inconnu! De même que Quaré et Aubert(in). D'autres noms en revanche renvoient à des trouvères ou à des personnages historiques que Pierre $\operatorname{Marot}^{30}$ et Maurice Delbouille ${ }^{31}$ ont pour la plupart identifiés. Presque tous les partenaires et juges gravitant autour de Roland de Reims avaient, sinon des liens directs, du moins des accointances avec la cour des comtes de Bar: Jehan de Bar (CLVIII; CLXII; CLXIV; CLXVIII) et Thibaud, son frère (CLXXI), Jacques de Longuyon (CLXII), Jean de Bayon (CLXIV), Burnequin de Riste (CLXIV), Habrant de Briey (CLXXXI), Gilles II d'Avocourt (CLXXXI), Raoul de Mercis (CLVIII), Jacques de Billi (CLVII, CLX, CLXVI), Mahaut de Commercy et la comtesse de Linange (Mahaut et Jeanne d'Aspremont) (CLVI et CLXVII), etc. Nous sommes en présence d'œuvres inscrites dans un contexte strictement lorrain, et de datation précise :« entre 1305 et 1316 » (Delbouille); « entre 1305 et septembre $1310 »$ (Jung). Et, cette foisci, les enseignements de la langue ne sauraient être suspectés : les jeux-partis du chansonnier I ne sont pas «lorrains», parce qu'un copiste leur aurait donné une coloration dialectale lorraine, mais parce qu'ils ont été composés et «joués» en Lorraine! Certes, des poètes de régions voisines ont pu collaborer au recueil, tel Jehan de Chison, sans doute originaire de Cysoing (CLXII); tel surtout Rolant de Rains. Mais qu'un Picard ou un Champenois aient trouvé en Lorraine une terre d'accueil où exercer leur art n'a rien de bien surprenant: Jacques Bretel, parent de Jean, n'a-t-il pas rimé pour la noblesse lorraine son Tournoi de Chauvency? Preuve

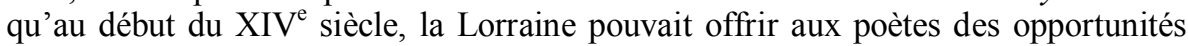
qu'ils ne trouvaient peut-être plus chez eux. Le cloisonnement régional n'est en tout cas pas une réalité médiévale: en amont de la sub-tradition de la sotte chanson

\footnotetext{
${ }^{29}$ A. Långfors, Deux recueils de sottes chansons, (op. cit.), p. 29.

${ }^{30} \mathrm{P}$. Marot, «Identifications de quelques partenaires et juges des 'unica' des jeux-partis du chansonnier d'Oxford", Bibliothèque de l'École des Chartes, 88, 1927, p. 266-274.

${ }^{31}$ M. Delbouille, «À propos des jeux-partis lorrains du chansonnier Douce $308(I)$ », Revue Belge de Philologie et d'Histoire, 12, 1933, p. 132-140.
} 
(réputée "picardo-artésienne») se trouve un autre Champenois : Robert de Reims, dit La Chèvre ! ${ }^{32}$

Tout s'accorde, comme l'écrit Maurice Delbouille, «pour attester l'existence d'un mouvement qui dut transporter de Picardie vers l'Est, dans la seconde moitié du XIII ${ }^{\mathrm{e}}$ siècle, une part de la culture littéraire qui s'était épanouie dans le cercle d'Arras aux environs de $1250 »^{33}$. Le Barrois tout spécialement paraît avoir été très tôt ouvert aux productions courtoises des autres régions d'oïl; dans un jeu-parti opposant Jean d'Arcis à Chardon de Croisille (CXLVII), Henri II de Bar était déjà appelé à juger en ces termes :

Hanris de Bar, qui cortoisie,

Et valor, et chevalerie,

Et amor maintient lëaulment

Proi k'en face lou jugement.

Cet hommage appuyé au seigneur lorrain inspira à Pierre Marot un commentaire des plus sensés :

Ainsi au XIII ${ }^{\mathrm{e}}$ siècle, la cour des comtes de Bar prisa la poésie. Il n'y a là rien que de naturel ; nous savons qu'à cette époque, les comtes de Bar étaient à l'apogée de leur puissance et étaient riches. Le Barrois était proche de la Champagne, les comtes avaient, en outre, de nombreuses possessions dans le nord de la France. Les poètes barrois n'eurent pas à aller loin pour chercher leurs modèles. ${ }^{34}$

C'est précisément dans l'entourage d'un seigneur de Bar, Jehan (mort en 1314), que les jeux-partis du groupe Roland de Reims ont été «desputés ». C'est également pour un commanditaire lorrain (barrois?) que le chansonnier $I$ a été composé et transcrit. Si l'identité dudit commanditaire reste un mystère, le manuscrit nous renseigne au moins sur le goût et sur l'attente du public de la région au début $\mathrm{du} \mathrm{XIV}^{\mathrm{e}}$ siècle. Or, ce goût, loin de dénoter quelque ringardise provinciale, se signale par sa totale perméabilité à la nouveauté. Marcel-René Jung a récemment mis l'accent sur ce trait : non seulement le compositeur du chansonnier $I$ a accordé sa faveur à des genres nouveaux (estampies, ballettes, sottes chansons...), mais encore, s'agissant de genres «anciens » tels que le grand chant ou le jeu-parti, à des types métriques neufs : «J'ai étudié - écrit Jung - la section du grant chant, et j'ai constaté que le choix des chansons courtoises obéit à une volonté de varier les formes métriques $»^{35}$. Même constat à propos des jeux-partis repris de sources variées (pièces 3.24 à $3.31 \mathrm{a})$ :

On constate d'abord qu'un seul des jeux-partis transcrits dans le manuscrit d'Oxford présente un front $a b b a$, bien que cette forme soit attestée dans une trentaine de jeux-

\footnotetext{
${ }^{32}$ Cf. W. Mann, «Die Lieder der Dichters Robert de Rains gennant La Chievre », Zeitschrift für romanische Philologie, 23, 1899, p. 79-115; spéc., p. 101-102, pièce $\mathrm{n}^{\circ} 5$.

${ }^{33}$ M. Delbouille, art. cit., p. 139.

${ }^{34}$ P. Marot, art. cit., p. 273-274.

${ }^{35}$ M.-R. Jung, art. cit., p. 388.
} 
partis, dont la plupart appartient au cercle de Jean Bretel. On remarque par contre que la collection offre tout un éventail de différents types de strophes, du septain au onzain, toutes isométriques. ${ }^{36}$

Ce sont toutefois les unica du groupe Roland de Reims qui, du point de vue de la variété métrique, présentent le plus d'originalité. Långfors avait autrefois noté que dans ce groupe les couplets se ramenaient de 6 à 5 ; soit déjà une innovation. Mais la variation est bien plus profonde ; elle porte, comme Jung l'a souligné, sur la multiplicité des types strophiques :

Les strophes des unica vont du septain au quinzain, une pièce en septains (3.3), trois en huitains $(3.7,11,18)$, quatre en neuvains $(3.8,15,19,21)$, en dizains $(3.1,4,5$, $10)$ et en onzains $(3.6,16,20,33)$, une en douzains $(3.17)$, six en treizains $(3.2,13$, $14,32,34,35)$, deux en quatorzains $(3.9,12)$ et une en quinzains $(3.36)$. Ainsi les strophes longues du chansonnier d'Oxford appartiennent toutes à des jeux-partis de l'entourage de Rolant. ${ }^{37}$

Même si la Lorraine est d'ordinaire regardée par les médiévistes comme une terre peu représentative de la modernité littéraire, il est clair que l'une des ambitions des poètes réunis autour de Roland de Reims a été de reprendre le flambeau à l' «avant-garde» arrageoise, à une époque où son étoile déclinait. Dans cette optique, les jeuX-partis CLXXIV et CLXXV seraient à considérer comme une tentative extrême pour relancer de l'intérieur, mais dans un style "résolument moderne", la vieille machinerie poétique du débat «courtois». Dédaignant l'expédient du casus loufoque, solution tiède à laquelle les autres jeux-partis parodiques conservés renvoient tous peu ou prou, Roland, Quaré et Aubert(in) ont choisi de déplacer le jeu vers la parodie absolue. Ce radicalisme les rapproche d'une certaine façon des fatrassiers ${ }^{38}$. Un parallèle serait du reste à faire du côté des effets d'intertextualité. Dans les Fatrasies d'Arras, les poètes se sont en effet amusés à "farcir» leurs strophes d'allusions littéraires: Girart de Roussillon, Ogier le Danoys, Vers de la Mort, Chanson d'Audin, Renart le Nouvel, Robin et Marion, Du Prestre et d'Alison, Aucassin et Nicolette, etc. ${ }^{39}$; tout un jeu citationnel, parfois explicite, parfois voilé, à l'usage des seuls «connaisseurs». Dans les jeux-partis CLXXIV et CLXXV, les citations devaient à coup sûr former pour le public un piment essentiel du plaisir. Mais pour que le public pût en jouir existait un préalable : qu'il connût le corpus de référence. Une question se pose dès lors : les sottes chansons du chansonnier $I$ (dont Långfors voulait absolument qu’elles fussent «picardo-artésiennes») ne seraient-

${ }^{36}$ M.-R. Jung, «À propos des jeux-partis du chansonnier I (Oxford, Bodl. Libr., Douce 308) », Miscellanea Medicevalia. Mélanges offerts à Philippe Ménard, études réunies par J.-C. Faucon, A. Labbé et D. Quéruel, Paris, Champion, 1998, t. I, p. 705-712 ; spéc., p. 711.

${ }^{37}$ M.-R. Jung, « Les formes strophiques... », art. cit., p. 389.

${ }^{38}$ Sur ce rapprochement, cf. M. Gally, « Poésie en jeu : des jeux-partis aux fatrassiers », Arras au Moyen Âge. Histoire et Littérature, textes réunis par M.-M. Castellani et J.-P. Martin, Arras, Artois Presses Université, 1994, p. 71-80 ; Parler d'amour au puy d'Arras. Lyrique en jeu, Orléans, Paradigme, 2004, p. 141-145.

${ }^{39}$ Cf. P. Uhl, op. cit, p. 26-36. 
elles pas tout bonnement lorraines ? Rien ne s'opposerait d'ailleurs à ce que Roland et ses deux confrères y aient mis la main. La parfaite imprégnation du corpus dont ils témoignent, l'agilité avec laquelle ils en jonglent (probablement dans les conditions d'une performance improvisée), la "faute commune» qu'ils partagent avec les chanteurs «sots» sur les exploits et les goûts dou boin conte Adegier (confondu dans la sotte chanson III et le jeu-parti CLXXV avec Turgibus), etc., tout le suggère.

Quoi qu'il en soit les pièces CLXXIV et CLXXIV forment pour la subtradition du jeu-parti une ponctuation forte. Dès l'origine, le genre a balancé entre orthodoxie courtoise et contestation de la doxa, mais toujours en préservant les formes. Même dans le cadre d'un débat «pour rire», le fonds idéologique de la «courtoisie» resta un bien commun; la référence implicite de tout questionnement d'amour. D'où l'ambiguïté foncière du genre, à la fois inscrit dans l'orthodoxie et critique par rapport à celle-ci :

Partimen et jeu parti - constate Michèle Gally - participent de l'invention d'un discours normatif courtois et démontrent au moins sa difficulté, au mieux son inanité. La norme n'est alléguée que pour être combattue par une autre, dénoncée comme un leurre ou un impossible. Le jeu-parti arrageois, dernier fleuron du genre, plus franchement ludique et nourri de registres non courtois et de registre multiples, confirme moins un code courtois qu'il n'en met en scène le dysfonctionnement. ${ }^{40}$

Les deux pièces parodiques lorraines du groupe Roland de Reims ne préservent, elles, plus du tout les formes, mais simplement la forme! Ce n'est plus, comme dans les autres jeux-partis parodiques, la virtuosité à débattre de tel ou tel cas burlesque qui motive le jeu, mais celle qui consiste à enfiler avec le plus grand naturel des bourdes énormes tirées du corpus de la sotte chanson contre Amour, tout en respectant le moule poético-discursif conventionnel du débat courtois. Par rebond, on liquide ici toute la «vieillerie poétique» (grans chans et jeu-parti confondus) ! C'est là la réponse (j'allais dire la revanche) «moderne» du petit cénacle - de la coterie $^{41}$ - réuni(e) à la cour de Jean de Bar autour de Roland de Reims.

Patrice Uhl

Université de La Réunion

\footnotetext{
${ }^{40} \mathrm{M}$. Gally, «Entre sens et non-sens : approches comparatives de la tenso d'oc et du jeu-parti arrageois ", Il genere tenzone nelle letterature romanze delle Origini, a cura di M. Pedroni e A. Stauble, Ravenna, Longo, 1999, p. 223-235 ; ici p. 234.

${ }^{41}$ Mot de P. Zumthor, op. cit. p. 43, pour désigner le milieu culturel restreint dans lequel se cultivaient jeux-partis et fatrasies.
} 